\title{
Genomic Biomarker
}

National Cancer Institute

\section{Source}

National Cancer Institute. Genomic Biomarker. NCI Thesaurus. Code C68753.

A measurable DNA or RNA characteristic that is an indicator of normal biologic processes, pathogenic processes, and/or response to therapeutic or other intervention. The definition for a genomic biomarker is not limited to human samples. A genomic biomarker could, for example, reflect: the expression of a gene; the function of a gene; the regulation of a gene. A genomic biomarker can consist of one or more deoxyribonucleic acid (DNA) or ribonucleic acid (RNA) characteristics. The definition for a genomic biomarker does not include the measurement and characterisation of proteins or low molecular weight metabolites. DNA characteristics include, but are not limited to: single nucleotide polymorphisms (SNPs); variability of short sequence repeats; DNA modification, e.g., methylation; insertions; deletions; copy number variation; cytog enetic rearrangements, e.g., translocations, duplications, deletions or inversions. RNA characteristics include, but are not limited to: RNA sequence; RNA expression levels; RNA processing, e.g., splicing and editing; microRNA levels (ICH Draft Consensus Guideline E15). 